\title{
MEDIOS ONLINE Y PUBLICIDAD. PERFILES PROFESIONALES EN EDUCACIÓN SUPERIOR
}

\author{
Natalia Papí-Gálvez: Universidad de Alicante. España \\ natalia.p@ua.es
}

Sonia López-Berna: Universidad de Alicante. España

sonia.lopez@ua.es

\section{RESUMEN}

Los cambios tecnológicos producidos en la sociedad de la información, con especial atención a Internet, inciden también en el sistema educativo. Esta situación es especialmente significativa en aquellas carreras con perfiles profesionales definidos y que utilizan los medios para llegar a sus públicos, como es el caso de la Publicidad y las Relaciones Públicas. Se plantea un estudio exploratorio que pretende averiguar si la actual oferta educativa integra las competencias relacionadas con los perfiles profesionales en torno al medio online. Se utilizan las definiciones y competencias de los perfiles centrados en el medio online (ej. Community Manager, SEO, SEM) y otras ampliamente conocidas por el sector publicitario (planificador en Internet). La investigación se centra en las cinco universidades españolas con más alumnos en estudios de publicidad y relaciones públicas: UCM, RJC, UVA, UA y UOC. Se analizan los cursos de licenciatura aún vigentes, títulos propios, masteres universitarios y doctorado. Todos ellos en relación directa con la publicidad y las relaciones públicas. Los resultados permiten evaluar tanto la información disponible como la oferta educativa para el aprendizaje de las competencias de estos perfiles en el marco de la EEES.

PALABRAS CLAVE: Internet - Perfiles Profesionales - Publicidad - EEES - Sociedad de la Información

\section{ON LINE MEDIA AND ADVERSTISING. PROFESSIONAL PROFILES}

\footnotetext{
${ }^{1}$ Autor correspondiente

Natalia Papí-Gálvez: Profesora Doctora Titular. Universidad de Alicante. Alicante (España).

Correo: natalia.p@ua.es
} 


\title{
IN HIGHER EDUCATION
}

\begin{abstract}
The technological changes in the information society, with special attention to the Internet, also affect the educational system. This is particularly significant in those degree studies with defined professional profiles that use the media to reach their audiences, such as the Advertising and Public Relations. This raises an exploratory study intended to find out whether the current educational offerings include skills related to professional profiles about online media. Definitions and competencies of profiles focused on online media (eg Community Manager, SEO, SEM) are used and other widely known by the advertising industry (online planner). The research focuses on the five Spanish universities with more students in studies of advertising and public relations: UCM, RJC, UVA, AU and UOC. We analyze the degree courses still in force, masters and doctorate. All of them are directly related to advertising and public relations. The results allow to evaluate both the information available as educational opportunities for learning the skills of these profiles in the framework of the European Space for Higher Education.
\end{abstract}

KEY WORDS: Internet - professional profiles - advertising - European Space for Higher Education - information society

\section{INTRODUCCIÓN}

\subsection{Internet como oportunidad de crecimiento}

Internet es el único medio cuya penetración ${ }^{2}$ ha crecido de forma constante durante 14 años, ofreciendo, así, una oportunidad de negocio en el sector publicitario. De hecho, es el único cuya inversión no ha caído con la crisis.

En 1997, este medio registraba una penetración del 0,9\% (AIMC, 2011). Su crecimiento fue exponencial durante los cuatro años siguientes, llegando casi a duplicar usuarios cada año. Desde 2002 hasta 2008 ha crecido anualmente del orden de 3 puntos porcentuales. En plena crisis, su aumento es aún mayor. Tanto en 2009 como en 2010 crece 4 puntos. También en el año 2011 ha seguido expandiéndose. Su penetración alcanza el 41,5\% de la población española, por encima de los Diarios (38\% según AIMC, de octubre 2010 a mayo 2011).

\footnotetext{
2 De toda la población de un país, la que es usuaria (consumidora o audiencia) de un medio
} 
En cuanto a la inversión publicitaria, Internet ha ido ganando terreno. En 2003, a Internet se le destina sólo un 1,3\% de toda la inversión de los medios considerados convencionales por Infoadex. En 2010 ya es del 13,5\%3. Es decir, en siete años, aumenta 12,2 puntos. Los peores años para la inversión publicitaria en los medios convencionales son 2008 y 2009. Todos los medios convencionales (sin Internet) descienden, en 2008, una media del $18 \%$ y, en 2009, del 25,6\%. En contrapartida, Internet aumenta un $26,5 \%$ en 2008, y un 7,2\% en 2009. Es más, en 2010 (último año disponible) Internet sigue ofreciendo datos muy favorables $(+20,7 \%)$ pese a la leve recuperación de otros medios tales como el cine, dominicales, radio, exterior y televisión.

Es más, en el propio medio, las fórmulas convencionales están siendo desbancadas por las menos convencionales. La inversión en buscadores y en enlaces patrocinados tiene incrementos mayores en todo el periodo (2003-2010) que la publicidad gráfica, superándola definitivamente en el año 2008. Su incremento anual también ha sido siempre mayor (Infoadex, 2011; Infoadex, 2008).

Y habría que considerar la búsqueda orgánica en buscadores, pues al no requerir un pago a cambio de la posición conseguida, no puede ser recogida como inversión publicitaria en Infoadex. A ello se le debe unir la explosión de los medios sociales y la posibilidad que estos ofrecen como vehículo de comunicación y oportunidad de negocio. De hecho, sólo en nuestro país, la participación en redes sociales por parte de los usuarios de Internet, en dos años (de 2008 a 2010), ha pasado de ser un fenómeno emergente a estar completamente consolidado (TCAnalysis, 20114 ). Hoy en día hablar de redes sociales es hablar de Facebook.

En abril de 2011, Facebook encabezaba la lista de las 1000 páginas más visitadas en todo el mundo (Google Doubleclick Ad Planner. www.google.com/adplanner/static/top1000/ ). Cuenta con más de 500 millones de usuarios. En España tiene aproximadamente 15 millones de usuarios (julio de 2011).

España es el quinto país con más usuarios en esta red de los 27 miembros de la Unión Europea (UE). Por encima están (en orden): Reino Unido, Francia, Italia y Alemania (www.facebook.com/advertising). En cuanto a la penetración ${ }^{5}$, España ocupa el puesto 17 (con un 31\%), levemente por debajo de la media de la UE (32\%). Únicamente dos países (UK, con 48\% y Malta con 60\%) quedan cerca o superan el $48 \%$ de Estados Unidos. Se comprueba, no obstante, cómo en España, sólo Facebook tiene una penetración similar a los diarios, por seguir con la referencia utilizada

\footnotetext{
${ }^{3}$ Se ha sacado el cálculo de los datos brutos proporcionados por Infoadex en sus resúmenes. La estimación publicada por Infoadex es algo más elevada (13,8\%).

${ }^{4}$ http:/ / www.tcanalysis.com/2011/03/22/infografia-sobre-el-uso-de-las-redes-sociales-en-espana/

${ }^{5}$ Dato propio. Cálculo: (Usuarios de Facebook/ población) X 100. Los usuarios de Facebook se han extraído de la página www.facebook.com/advertising el 4 de julio de 2011. Se ha incluido toda la población del país sin otros criterios de segmentación. La población se ha extraído de dos fuentes. Para los países europeos, proporciona el dato Eurostat, recogido a 1 de enero de 2011. Para EEUU, la กNIt t al dato ac do m1ก
} 
también para Internet.

La gran oportunidad de negocio que ofrece Facebook radica tanto en la millonaria cantidad de usuarios que participan como en la información que tiene de ellos. Estas particularidades hacen de esta plataforma un soporte internacional para la Publicidad y para la Relaciones Públicas y una base de datos única para las acciones de marketing, aunque Facebook no es el único medio social. A este respecto, los medios sociales son:

(...) un grupo de aplicaciones basadas en Internet que se desarrollan sobre los fundamentos ideológicos y tecnológicos de la Web 2.0, y que permiten la creación y el intercambio de contenidos generados por el usuario (traducido de Kaplan y Haenlein, 2010,p. 61).

En consecuencia, además de las redes sociales como Facebook, estos autores consideran medios sociales los blogs, los mundos virtuales sociales (i.e. Second Life), los proyectos de colaboración (i.e. Wikipedia), los agregadores de contenidos (i.e. Youtube) y los mundos virtuales del juego (i.e. World of Warcraft). Para la IAB, los medios sociales también son un conjunto de plataformas digitales. Esta asociación, en concreto, nombra los siguientes: "Blogs, Fotoblogs, Microblogs, Redes Sociales, Utilidades Gráficas, Redes Profesionales, Mundos Virtuales, Dating, Agregadores de Contenidos y, en general, cualquier soporte que ofrezca a sus usuarios la posibilidad de generar un contenido susceptible de ser compartido" (IAB, (s/a),p. 6).

Cada plataforma o aplicación considerada tiene atributos que los hacen diferentes en términos de comunicación. Aunque, por ejemplo, Facebook comparte con otros medios sociales la posibilidad de abrir canales de comunicación más próximos al público objetivo, adquirir feedback inmediato y desarrollar estrategias menos intrusivas que las convencionales $\left(\mathrm{IAB},(\mathrm{s} / \mathrm{a})^{6}\right)$ si se gestiona adecuadamente. En este sentido, Kaplan y Haenlein (2010) proporcionan diez recomendaciones clasificándolas según las dos dimensiones que tienen los medios sociales, es decir, cinco centradas en el uso de los medios y otras cinco correspondientes a su componente social (ser social).

El fenómeno de los buscadores tiene una trayectoria similar. Google se funda en 1998 y en poco tiempo desbanca a Altavista. Como empresa se instala en España en el año 2003, momento en el que Altavista es adquirida por Yahoo! y parte del mercado español ya estaba utilizando Google. En 2007 Google compra DoubleClick, haciendo evidente la potencialidad (y la demanda) de la plataforma para la publicidad.

\footnotetext{
${ }^{6}$ IAB (s/a): Cuadernos de comunicación interactiva. Vol. 8. Libro Blanco de la IAB. La comunicación
} en medios sociales, Madrid: Edipo. 
Actualmente Google es el principal motor de búsqueda. Tiene el 90\% del mercado internacional y el 96\% del nacional. Muy de lejos le siguen otros como Bing o Yahoo!, prácticamente inexistentes cuando se habla de España y de Europa (http://gs.statcounter.com/\#search_engine-ww-yearly-2008-2011).

No obstante, desde los primeros buscadores con cierta repercusión (i.e. Altavista) las firmas y empresas con páginas webs se dieron cuenta de lo importante que era estar en los primeros puestos de un resultado de búsqueda. Así, se puede considerar que los Webmasters de entonces son los antecesores de los SEOs de ahora, que adaptados a la tecnología del momento, entre otras tareas, tenían la voluntad de posicionar la web en el buscador. Google, como principal buscador, establece las reglas de juego en la optimización.

Estos datos explican lo que en el terreno laboral está ocurriendo. Los profesionales perciben que hay una gran demanda de puestos especializados en Internet y en los medios sociales (IAB-SPAIN, 2010) ${ }^{7}$.

Los medios sociales siguen ocupando las primeras posiciones en el estudio de junio de 2011 (IAB-SPAIN, 2010). En este último informe se recoge ya de forma explícita a los community managers, como primer perfil, seguido de otros estrategas en estas aplicaciones. Según este informe además:

Sin salir del ámbito digital, destaca la demanda genérica de responsables de marketing, comunicación o publicidad especializados en esta área (19\%). Este dato deja entrever que el mercado laboral para estos profesionales más generalistas aún no ha tocado techo. Otros perfiles del ámbito tecnológico que son mencionados de forma destacada son: expertos en SEO, SEM y posicionamiento Web (12\%), desarrolladores Web y de aplicaciones (11\%) y expertos en analítica Web (7\%)", (IAB-SPAIN, 2011,p. 27).

En consecuencia, los perfiles que se podrían destacar según estos estudios en el ámbito de la Publicidad y de las Relaciones Públicas son: los genéricos relacionados con la publicidad en Internet (también a través de dispositivos móviles) y perfiles más concretos como el gestor de comunidades (Community Manager), SEM/SEO.

Todo lo anterior queda de alguna forma resumido por la idea que AERCO transmite de este medio: “Internet no es un medio más. Es una infraestructura sobre la que construir medios de comunicación (...) No todo el que está en Internet, es susceptible

\footnotetext{
7 SMO (Social Media Optimization), Social Media Marketing, Redes Sociales, Web 2.0 y 3.0, Cloud Computing (29\% de respuestas); Mobile / Mobile Mkt / Mobile Advertising (13\%); Internet / Entorno Digital /Tecnología en general (4,4\%); Programación (Ajax, HTML,flash, aplicaciones, etc) /Desarrollo Web (4,4\%); Gestión de comunidades /Community Management(4\%); Marketing / Publicidad digital(4\%); SEO /SEM/ Buscadores/ Search (4\%).
} 
de recibir impactos publicitarios." (AERCO, 2009,p. 4). Y, por ello, son necesarias estrategias de comunicación adecuadas y los profesionales para llevarlas a cabo.

\subsection{Los perfiles profesionales en Internet: viejos y nuevos conceptos}

\section{Community Manager}

Según AERCO (2009) el Community Manager (CM) ${ }^{8}$ es un gestor de la parte "social" del medio $^{9}$, cuyo antecesor es el moderador de las comunidades on-line. La Asociación asegura, por tanto, que existen "principalmente dos tipos de CM: el gestor de comunidades online "ad-hoc» y el gestor de la comunidad de la marca" (AERCO, 2009,p.3). En Publicidad y Relaciones Públicas interesa especialmente el segundo perfil. Según esta asociación, el CM es:

(...) aquella persona encargada o responsable de sostener, acrecentar y, en cierta forma, defender las relaciones de la empresa con sus clientes en el ámbito digital, gracias al conocimiento de las necesidades y los planteamientos estratégicos de la organización y los intereses de los clientes (...) buscan necesariamente crear o mantener comunidad en torno a algo o alguien", - la diferencia con otros perfiles como el social media analyst es que el punto de vista de este segundo es - "más corporativo y menos de usuario. (...) El verdadero potencial está en establecer una relación de confianza con la comunidad de usuarios o simpatizantes de la marca, recoger el feedback de los mismos y utilizarlo para proponer mejoras internas." (AERCO, 2009, p. 5$6)$.

Se comprueba que aunque el CM tiene tareas más especializadas le correspondería desarrollar, de acuerdo con la anterior explicación, por ejemplo, <<destrezas para identificar, clasificar y comunicarse eficazmente con los diferentes públicos $>>$, que se trata de la competencia profesional «E19» recogida en el Libro Blanco de las titulaciones de comunicación para los perfiles de Publicidad y Relaciones Públicas. En este sentido, es en el perfil profesional denominado Gestor de Comunicación Corporativa donde se describen destrezas profesionales vinculadas a las competencias del CM.

\footnotetext{
8 "Profesional responsable de la gestión estratégica de la imagen y de la comunicación corporativa, tanto en su naturaleza intangible (identidad visual, comunicación y cultura corporativa) como en sus interrelaciones funcionales (financiera, comercial, de producción, etc.), y de establecer diálogos constructivos con los diferentes públicos relevantes de la empresa, internos y externos. También se ocupa de la reputación corporativa, en función del grado de cumplimiento de sus compromisos en relación con los públicos implicados". (ANECA, 2004: 272).

9 Kaplan y Haenlein (2010), a este respecto son claros cuando enumeran las 10 recomendaciones dirigidas a una firma que pretenda utilizar los medios sociales. En la dimensión de Medios quedan decisiones del tipo: elección de los soportes y de otros medios (sociales, tradicionales...), elección de la aplicación específica de la acción o creación de la misma, combinación de medios sociales, mensajes no ambiguos, públicos que pueden acceder. Las sociales son del tipo: ser activo, ser interesante, ser familiar, ser honesto, ser humilde...
} 
Ahora bien, y aunque el CM debe poseer un conocimiento especializado de las aplicaciones que permitan generar conversación entre el público, y debe centrarse únicamente en el medio online (tabla 1), podría encuadrarse igualmente en las competencias normalmente vinculadas a las Relaciones Públicas. De hecho, las nueve características de las Relaciones Públicas enumeradas por Castillo (Castillo, 2010) podrían ser adaptadas, todas ellas, a la actividad específica del $\mathrm{CM}^{10}$.

Desde la perspectiva de las Relaciones Públicas ${ }^{11}$ se entiende que es fundamental atender a las demandas de los públicos, de manera que se dé siempre una comunicación bidireccional, en ambos sentidos, que posibilite el entendimiento mutuo; consideración que el CM hace propia y la lleva a la práctica en un entorno concreto.

\footnotetext{
10 a). Actúan de acuerdo con un programa planificado y sostenido como parte de la dirección de la organización; b). Tratan de las relaciones entre una organización y sus públicos; c). Supervisan el conocimiento, opiniones, actitudes y comportamiento dentro y fuera de la organización; d). Analizan el impacto de estrategias, procedimientos y acciones sobre los públicos; e).Ajustan aquellas estrategias, procedimiento y acciones que puedan estar en conflicto con el interés público o la supervivencia de la organización; f).Aconsejan a la dirección sobre el establecimiento de nuevas estrategias, procedimientos y acciones que puedan ser mutuamente beneficiosas para la organización y sus públicos; g.) Establecen y mantienen comunicación bidireccional entre la organización y sus públicos; h). Realizan cambios específicos en el conocimiento, opiniones, actitudes y comportamientos dentro y fuera de la organización; i). Impulsan nuevas relaciones o mantienen las existentes entre una organización y sus públicos (Castillo, 2010)

11 Para conseguir los objetivos, las Relaciones Públicas deben controlar el cauce de comunicación con todos los públicos con los que ésta se relaciona, tanto a nivel interno (personal, proveedores, accionistas...) como a nivel externo (clientes). Pone el acento fundamentalmente en la comunicación interna (empleados), en la gestión de crisis y en la reputación social corporativa (diferencias esenciales con la Publicidad). Se trata, pues, de construir mensajes cuyo protagonista principal sea la entidad que promueve la acción de comunicación, su filosofía empresarial o su política de empresa,

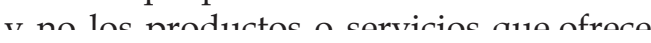


Tabla 1. Competencias del Community Manager Fuente: Adaptado de AERCO (2009)

\begin{tabular}{|c|c|c|}
\hline Aptitudes técnicas & Habilidades sociales & Actitud \\
\hline $\begin{array}{l}\text { Conocimiento sectorial. } \\
\text { Conocimientos de } \\
\text { marketing, publicidad y } \\
\text { comunicación } \\
\text { corporativa. } \\
\text { Redacción. Creatividad } \\
\text { (en la economía de la } \\
\text { atención y } \\
\text { de la sobreabundancia } \\
\text { de la información) } \\
\text { Experiencia en }\end{array}$ & $\begin{array}{l}\text { Buen conversador } \\
\text { (saber escuchar, saber } \\
\text { responder) } \\
\text { Resolutivo (da } \\
\text { respuesta de forma } \\
\text { rápida y adecuada) } \\
\text { Agitador (incentiva la } \\
\text { participación) } \\
\text { Empático } \\
\text { Asertivo (tiene } \\
\text { carácter y } \\
\text { personalidad propios) }\end{array}$ & $\begin{array}{l}\text { Innovación (abierto a } \\
\text { probar aplicaciones y } \\
\text { servicios) } \\
\text { Útil (ser de ayuda) } \\
\text { Abierto (entiende y aprecia } \\
\text { la diversidad) } \\
\text { Accesible (es cercano en el } \\
\text { trato y vive con conexión } \\
\text { permanente o frecuente a } \\
\text { la red) } \\
\text { Conector (detecta y facilita } \\
\text { oportunidades, conectando }\end{array}$ \\
\hline $\begin{array}{l}\text { comunicación online } \\
\text { (conocer los canales más } \\
\text { adecuados y tener } \\
\text { buenos } \\
\text { contactos en Internet). } \\
\text { Cultura } 2.0 \text { (normas y } \\
\text { valores) }\end{array}$ & $\begin{array}{l}\text { Comprensivo (valora } \\
\text { las opiniones del } \\
\text { resto) } \\
\text { Trabajo en equipo } \\
\text { Cabecilla (liderar y } \\
\text { saber } \\
\text { encontrar líderes) } \\
\text { Moderador (se } \\
\text { esfuerza por mantener } \\
\text { un ambiente cordial) } \\
\text { Incentivador (plantea } \\
\text { incentivos a los } \\
\text { usuarios } \\
\text { y detecta las carencias } \\
\text { en la comunidad) }\end{array}$ & $\begin{array}{l}\text { a miembros de la } \\
\text { comunidad entre sí) } \\
\text { Cazador de tendencias. } \\
\text { Evangelista (apasionado de } \\
\text { la marca, de } \\
\text { la empresa y de la vida) } \\
\text { Defensor de la comunidad } \\
\text { (representa a los } \\
\text { clientes y usuarios ante la } \\
\text { empresa) } \\
\text { Transparente (en las } \\
\text { normas y en la igualdad } \\
\text { entre los usuarios) }\end{array}$ \\
\hline
\end{tabular}




\section{SEO/SEM}

SEO son las siglas en inglés de "optimización de motores de búsqueda" (Search Engine Optimization) o de "optimizador de motores de búsqueda" (Search Engine Optimizer)(http://www.google.com/support/webmasters/bin/answer.py?hl=es\&a $\underline{\text { nswer }=35291})$. De acuerdo con la IAB $\left(2010^{12}\right)$ :

SEO (Search Engine Optimization) es la práctica de utilizar un rango de técnicas, incluidas la reescritura del código html, la edición de contenidos, la navegación en el site, campañas de enlaces y más acciones, con el fin de mejorar la posición de un website en los resultados de los buscadores para unos términos de búsqueda concretos (...) (IAB- SPAIN, 2010,p. 9).

Según Google:

(http://www.google.com/support/webmasters/bin/answer.py?hl=es\&answer=3529 1), los servicios que puede ofrecer un SEO o una agencia o consultoría vinculados a esta figura son: revisión del contenido y la estructura del sitio, asistencia técnica en el desarrollo de sitios web (por ejemplo, alojamiento, redireccionamientos, páginas de error, uso de JavaScript, etc.), desarrollo de contenidos, administración de campañas de desarrollo empresarial online, investigación sobre palabras clave, formación en SEO y experiencia en sectores específicos y regiones geográficas.

Todos ellos persiguen la optimización de la web según parámetros acordados previamente (palabras clave, ámbitos geográficos...) con la intención de que aparezca en los primeros puestos de una búsqueda orgánica (principal función del SEO) y la web se haga visible ante la competencia. A este respecto, se debe diferenciar de la publicidad de pago en buscadores (SEM) a través de enlaces patrocinados. En realidad, se trata de dos estrategias comerciales distintas que pueden desarrollarse por profesionales con perfiles diferentes, aunque será necesaria la colaboración entre ellos así como su integración en la estrategia de negocio y de comunicación de la empresa.

De hecho, el SEO debe comprender cómo funciona el algoritmo de búsqueda de los buscadores y tener un conocimiento profundo de la elaboración y diseño (no estético) de las webs. Por ello, debe conocer las tecnologías que pueden ser reconocidas por los buscadores y rehacer, si fuera necesario, la página. Debe saber trabajar con archivos sitemaps, robots.txt, generar una estructura de enlaces internos, cambiar los contenidos de la propia web para adecuarlos a las diferentes tipologías de búsqueda, combinar el contenido con las etiquetas html, garantizar la rápida respuesta del servidor, saber interpretar los resultados de las estadísticas de la web, integrar adecuadamente las técnicas onside con las offside y poseer, en definitiva, otros

12 IAB (2010): Cuadernos de comunicación interactiva. Vol. 11. El libro blanco de IAB. SEO: optimización de wehs nara hisscadores. Buenas nrácticas v resıltados. I11lo de 2010. Madrid: IAB. 
conocimientos técnicos relativos a la web y a Internet que no en pocas ocasiones transciende los conocimientos generales del publicitario o relaciones públicas.

En cuanto al SEM, o Search Engine Marketing, éste es un perfil más general que responde a la promoción y aparición en buscadores. De acuerdo con Google (http://google.dirson.com/posicionamiento.net/sem/) el perfil SEM debe: controlar la oferta de servicios "de pago" que existen en los buscadores, conocer y poder elegir los sitios web en los que debe anunciarse una firma (los soportes); conocer los sitios en los que permiten la publicidad contextual y valorar su contratación; elegir las palabras clave que deben ser "compradas", el presupuesto necesario y disponible, el texto a incluir y la segmentación a utilizar. Se aprecia, por tanto, que este perfil está más cerca de lo que se conoce como un estratega publicitario, quien debe atender a las recomendaciones efectuadas desde la planificación de medios publicitarios.

\section{3 Perfiles genéricos especialistas en Internet}

Por una parte, cuando se solicitan perfiles genéricos especialistas en Internet, se está solicitando un conocimiento completo del medio. Se tratan de profesionales que entienden y conocen las posibilidades que proporciona el medio a nivel comercial y publicitario y, por tanto, pueden tomar decisiones relacionadas con varios tipos de campañas, incluidas en aquellas que se precisan las tareas desarrolladas por el Community Manager y el SEO/SEM.

La publicidad en Internet tiene como principal herramienta la página web y su contenido. Por tanto, los buscadores, los medios sociales, los móviles, las propias páginas web, son sólo soportes para llevar a cabo diferentes estrategias, campañas y formatos, tales como: búsqueda orgánica, enlaces patrocinados, publicidad contextual, publicidad gráfica (display), marketing de afiliación, advergaming (publicidad dinámica y estática), marketing móvil (campañas push y pull, y formatos publicitarios en móviles), email marketing, reputación online...

En este sentido, sería válida la clasificación de perfiles profesionales recogida en el Libro Blanco de las Titulaciones de Comunicación. Se advierte, también en esta ocasión, diferencias entre los estrategas, los encargados de la codificación del mensaje (creativos) y los investigadores y planificadores de medios. Por supuesto, todos ellos deben saber lo que significa hacer publicidad en Internet y tener los conocimientos y las capacidades para su realización. No obstante, por otra parte, estos perfiles han sufrido una especialización casi absoluta. En cuanto a los investigadores de medios, destaca, por ejemplo, el trafficker. 


\subsection{La reforma de los estudios universitarios}

El Ministerio de Educación, Cultura y Deporte, recogía claramente la necesidad de enfocar los nuevos grados a las demandas profesionales. El Documento Marco para la Integración del Sistema Universitario Español en el EEES lo expone de la siguiente forma:

Los objetivos formativos de las enseñanzas oficiales de nivel de grado tendrán, con carácter general, una orientación profesional, es decir, deberán proporcionar una formación universitaria en la que se integren armónicamente las competencias genéricas básicas, las competencias transversales relacionadas con la formación integral de las personas y las competencias más específicas que posibiliten una orientación profesional que permita a los titulados una integración en el mercado de trabajo. (MECD, 2003,p. 7- 8).

Esta consideración se formalizada en el Real Decreto 1393/2007, de 29 de octubre, por el que se establece la ordenación de las enseñanzas universitarias oficiales. En este sentido, la estructura general de las enseñanzas universitarias contempla, además de los grados, los Másters, que permitirían, a priori, una mayor especialización profesional:

Las enseñanzas de Master tienen como finalidad la adquisición por el estudiante de una formación avanzada, de carácter especializado o multidisciplinar, orientada a la especialización académica o profesional, o bien a promover la iniciación en tareas investigadoras." (MEC, 2007, Real Decreto 1393/2007, BOE n 260 del 30 de octubre de 2007, p. 44039)

Por tanto, al amparo de la filosofía de Bolonia, las competencias profesionales deben contemplarse en los planes de estudio de los grados universitarios y pueden ser consideradas en estudios posteriores, como los Masters.

Esta situación es especialmente significativa en aquellas carreras con perfiles profesionales definidos y que utilizan los medios para llegar a sus públicos, como es el caso de la Publicidad y las Relaciones Públicas.

El planteamiento español en materia de comunicación social, expuesto en el libro blanco de la ANECA, se sustenta en la existencia de una demanda profesional diferenciada para cada una de las titulaciones en comunicación ${ }^{13}$.

\footnotetext{
${ }^{13}$ Este enfoque difiere del sostenido en otros países de Europa, tales como Francia o el Reino Unido, en los que se ofertan títulos más generalistas, enmarcados bajo el epígrafe de "Estudios de comunicación", "Información y Comunicación" ó "Comunicación social" entre otros.
} 
Es decir, se defiende la existencia de tres títulos de grado, manteniendo que cada uno de ellos deberá diseñarse teniendo en cuenta las competencias profesionales que necesitan adquirir los futuros titulados, y no pudiendo establecerse títulos cuyos contenidos y efectos profesionales sean coincidentes, de manera que cada diploma deberá remitir en exclusiva a una determinada condición profesional (ANECA, 2004).

Se hace, así, necesario determinar qué funciones concretas en el ámbito de la comunicación social son las que realiza un Periodista, un Publicitario y/o Relaciones públicas y un titulado en Comunicación Audiovisual (López-Berna, PapíGálvez y Martín-Llaguno,2010).

De este modo, se diferencian cuatro perfiles generales vinculados a la Publicidad y Relaciones Públicas (ANECA, 2004):

a) Director de comunicación, investigador y estratega;

b) Investigadores, planificadores y compradores de medios;

c) Creativos/diseñadores y

d) Gestor de la comunicación corporativa. Igualmente se listan una serie de competencias de diferentes características (conocimientos disciplinares, competencias profesionales y académicas) estrechamente vinculadas a estos perfiles.

Se comprueba, como reflejo del año en el que se elaboró el Libro Blanco, que sólo el perfil "Creativos/diseñadores" incluye en su definición alguna mención a Internet. Además, lo hace de forma muy restringida, al recoger únicamente el Webmaster y asociarlo a la creatividad de la estructura de la Web.

Sin embargo, como se ha expuesto, dados los cambios tecnológicos producidos en la sociedad de la información, fundamentalmente vinculados a Internet, y tras el año 2004 (fecha en la que se publica el libro blanco), han aparecido y consolidado nuevos perfiles profesionales cuyas competencias específicas deberían poder incorporarse a la enseñanza universitaria.

En el curso 2010-2011 no se podía ofertar plazas de nuevo ingreso en el primer curso para las titulaciones a extinguir. En consecuencia, la elaboración de dichos planes se pudo realizar, aproximadamente, entre la publicación del Real Decreto 1393/2007, en octubre de 2007, hasta finales del año 2009, en todo caso, cuando la tendencia en el mercado español con respecto a las oportunidades que ofrecía el medio Internet era evidente.

Por tanto, este estudio pretende averiguar si la actual oferta educativa proporciona información suficiente sobre las salidas profesionales e integra las competencias relacionadas con los perfiles profesionales en torno al medio online. Así, se persigue: valorar la cantidad de información pública en las webs de las universidades sobre las salidas profesionales e identificar las competencias relacionadas con estas profesiones 
según tipo y nivel de estudio.

\section{METODOLOGÍA}

Se plantea un estudio exploratorio de la oferta educativa a través de las webs de las universidades.

La oferta educativa seleccionada incluye el grado en Publicidad (y Relaciones Públicas) y los cursos de licenciatura aún vigentes en esta especialidad. También se revisarán los títulos propios (cursos de especialista, experto y magisters), los masteres universitarios y los doctorados que tengan una relación directa con la publicidad y las relaciones públicas y con los departamentos vinculados a esta área en cada universidad.

La investigación se centra en las cinco universidades españolas con más alumnos en estudios de Publicidad y Relaciones Públicas. Según últimos datos del INE, curso 2009-2010, son: Universidad Complutense de Madrid, Rey Juan Carlos, Valladolid, Alicante y Oberta de Cataluña (tabla 2). Los alumnos de estas universidades suponen el 44,5\% de los matriculados en Publicidad y Relaciones Públicas de toda España.

Tabla 2. Alumnos matriculados curso 2009/ 2010

Fuente: Elaboración propia a partir de los datos del INE. Últimos datos disponibles.

\begin{tabular}{|c|c|c|c|}
\hline \multicolumn{2}{|c|}{ Universidades públicas } & \multicolumn{2}{|c|}{ Universidades privadas } \\
\hline Complutense de Madrid & 1759 & Oberta de Catalunya & 1035 \\
\hline Rey Juan Carlos & 1367 & San Pablo-CEU & 524 \\
\hline Valladolid & 1307 & Cardenal Herrera-CEU & 424 \\
\hline Alicante & 1058 & Ramón Llull & 373 \\
\hline Sevilla & 749 & Pontificia de Salamanca & 333 \\
\hline Málaga & 659 & Abat Oliba-CEU & 305 \\
\hline País Vasco & 584 & Navarra & 246 \\
\hline Barcelona & 436 & Vic & 240 \\
\hline Jaume I de Castellón & 431 & Francisco de Vitoria & 162 \\
\hline Vigo & 371 & San Jorge & 159 \\
\hline Autónoma de Barcelona & 358 & Europea de Madrid & 113 \\
\hline Rovira i Virgili & 292 & Antonio de Nebrija & 91 \\
\hline
\end{tabular}




\begin{tabular}{|c|c|c|c|}
\hline Murcia & 281 & $\begin{array}{l}\text { Católica S. Antonio de } \\
\text { Murcia }\end{array}$ & 88 \\
\hline Cádiz & 237 & $\begin{array}{l}\text { Europea Miguel de } \\
\text { Cervantes }\end{array}$ & 70 \\
\hline Girona & 200 & Camilo José Cela & 69 \\
\hline Miguel Hernández de Elche & 182 & & \\
\hline Pompeu Fabra (1) & 156 & & \\
\hline UNIV. PÚBLICAS & 10427 & UNIV. PRIVADAS & 4232 \\
\hline
\end{tabular}

Para dar respuesta al objetivo, se hablará de "competencia" en sentido amplio, según la definición del Proyecto Tuning ${ }^{14}$. Por lo que podrían aparecer las competencias sin necesidad de que estén formuladas como tales o estén incluidas como objetivos formativos.

No obstante, en la identificación de las competencias de los perfiles de Internet se tendrán en cuenta, para los perfiles genéricos, las del Libro Blanco de las Titulaciones de Comunicación aunque orientadas al medio Internet $\mathrm{y}$, para los perfiles especializados, las definiciones de las ocupaciones específicas, ambas recogidas en la introducción. La información que se analiza corresponde al curso 2011-2012, salvo en aquellos casos que no esté aún actualizada, por lo que se analizará la del curso 20102011. La información se recoge con las siguientes orientaciones por universidad y por tipo de oferta educativa (Tabla 3).

14 “las competencias representan una combinación de atributos (con respecto al conocimiento y sus aplicaciones, aptitudes, destrezas y responsabilidades) que describen el nivel o grado de suficiencia con

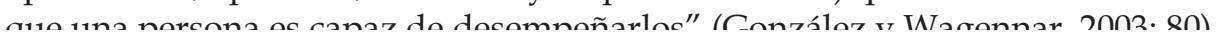


Tabla 3. Información relevante por objetivo

Objetivo 1. Valorar la cantidad de información pública en las webs de las universidades sobre el sector profesional.

Si se nombra el mercado laboral, el sector profesional, los perfiles o/y salidas profesionales en la oferta educativa (grados-licenciatura, masters, doctorados y cursos).

Por tanto, se busca la información en tres apartados:

- En la "información general" del grado-

licenciatura/master/doctorado/cursos

- En el nombre (plan de estudios) de las asignaturas (TODAS)

(módulos, líneas) del grado-licenciatura/master/doctorado/cursos.

- En la descripción o temario de las asignaturas del grado-licenciatura

(master/doctorado/cursos) QUE SEAN FUNDAMENTALES,

BÁSICAS, TRONCALES U OBLIGATORIAS

Si se nombran perfiles y si son generales (para publicidad y relaciones públicas) y/o específicos (para el medio Internet).

Si se definen los perfiles.

Si se exponen las competencias de tales perfiles.

Objetivo 2. Identificar las competencias relacionadas con estas profesiones según tipo y nivel de estudio.

Sólo se considera para este apartado la información que aparezca relativa a Internet. Se busca información relacionada con los perfiles generales (estrategas, creativos, investigadores/planificadores, relaciones públicas/corporate) o especializados (CM, SEO, SEM, Móvil, otros). Se recogerá, no obstante, toda mención a Internet o a tareas relacionadas con este medio. 
Finalmente, tras una primera fase de exploración, la muestra analizada queda en 40 tipos de oferta educativa (Tabla 4) distribuida por universidad como se recoge en el cuadro 4.

Tabla 4. Tipo y número de ofertas de estudio analizadas

\begin{tabular}{|c|c|c|c|}
\hline & & Frecuencia & Porcentaje \\
\hline Válidos & LICENCIATURA & 5 & 12,5 \\
\hline & GRADO & 5 & 12,5 \\
\hline & MASTER OFICIAL & 3 & 7,5 \\
\hline & MASTER & 11 & 27,5 \\
\hline & EXPERTO & 7 & 17,5 \\
\hline & POSTGRADO- & & \\
\hline & SINDEFINIR & & \\
\hline & OTROS & 1 & 2,5 \\
\hline & Total & 40 & 100,0 \\
\hline
\end{tabular}

Tabla 5. Ofertas analizadas por Universidad

\begin{tabular}{|l|r|r|}
\hline & Frecuencia & Porcentaje \\
\hline Válidos COMPLUTENSE & 14 & 35,0 \\
MADRID & 8 & \\
REY JUAN & 20,0 \\
CARLOS & 7 & 5,0 \\
VALLADOLID & 9 & 17,5 \\
ALICANTE & & 22,5 \\
OBERTA DE & 40 & 100,0 \\
CATALUNA & & \\
Total & & \\
\hline
\end{tabular}




\section{ANÁLISIS Y DISCUSIÓN}

\subsection{Valoración de la cantidad de información}

En cuanto a la cantidad de información disponible se observa que en todos los casos salvo en uno (grado de la Universidad de Valladolid) hay una introducción explicando de forma normalmente resumida el objeto o tema sobre el que gira la oferta educativa.

También hay información de los planes de estudio. Únicamente, y de forma excepcional, al estar compuesto de dos cursos de especialista, el Máster de la Universidad de Alicante no dispone de un plan de estudios detallado propio. Se trata de la suma de los cursos mencionados.

Se detecta, con todo, una clara carencia en los temarios de las asignaturas. El 58\% de los estudios analizados no disponen de los mismos. En este porcentaje se encuentran fundamentalmente aquellos estudios que son Títulos Propios de las Universidades. No obstante, también se echa en falta mayor información sobre los contenidos de las asignaturas del grado de los cursos que aún no han sido impartidos.

En cuanto a la información del sector, el 55\% de la oferta educativa hace alguna mención al respecto. Se observan más licenciaturas con este tipo de datos que en el resto de estudios, a excepción del único curso de formación continua incluido en "otros" (figura 1). Se debe destacar que los tipos de oferta educativa planteada desde un enfoque de competencias profesionales (grado, máster oficial, másters, cursos de expertos, otros postgrados) son las que menos menciones al sector hacen.

Igualmente el 55\% de la oferta educativa analizada nombra algún perfil (sea general, específico o los dos). En esta ocasión serán los grados los que recojan este tipo de información.

Destaca la ausencia de esta información en los títulos propios, incluso por encima de las licenciaturas (figura 2). Se comprueba cómo los perfiles específicos se concentran más en otro tipo de estudios que no son la licenciatura (figura 3) en especial en los grados y en los másters (que son títulos propios). 


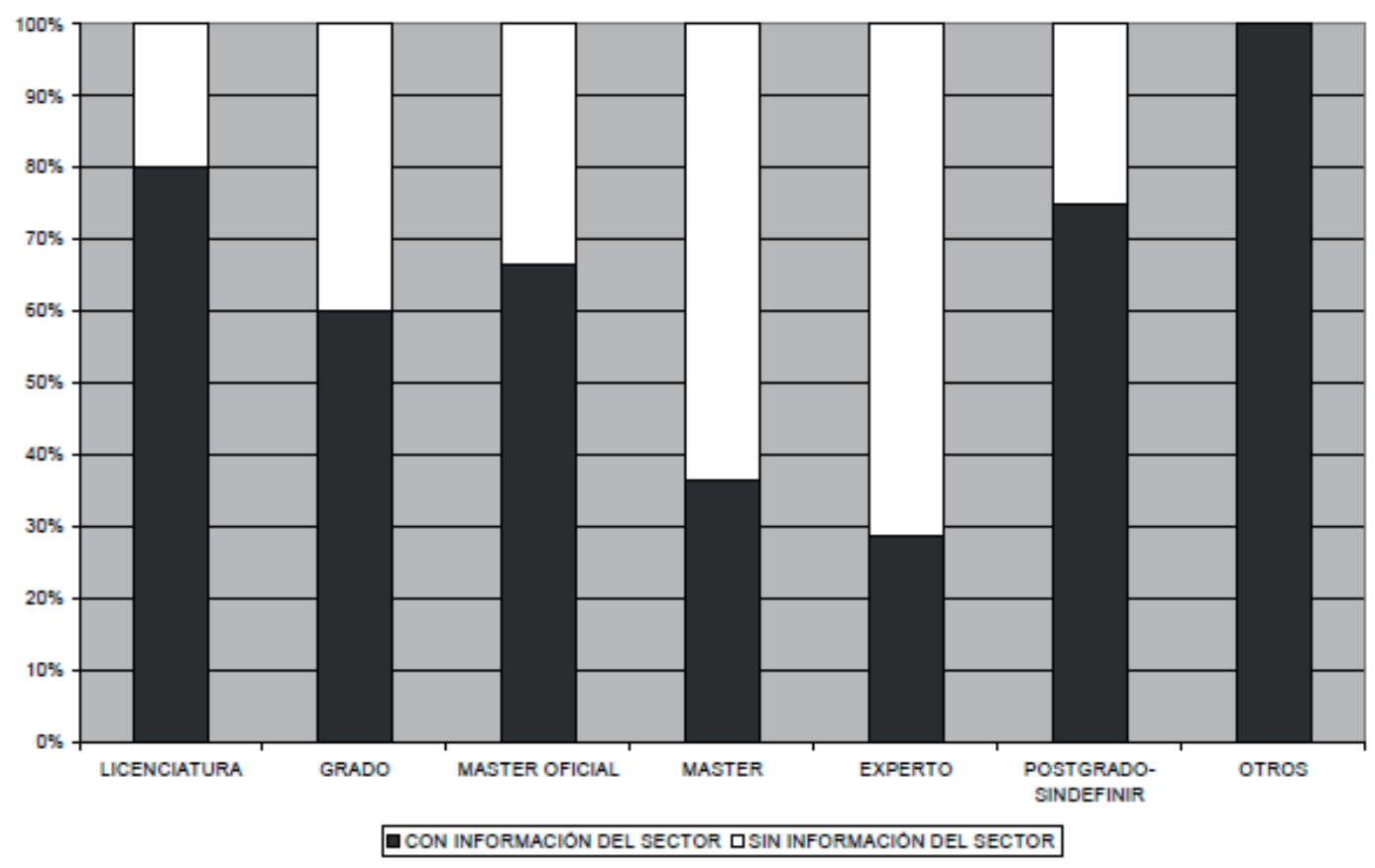

Figura 1. Información sobre el sector profesional o laboral según tipo de oferta educativa

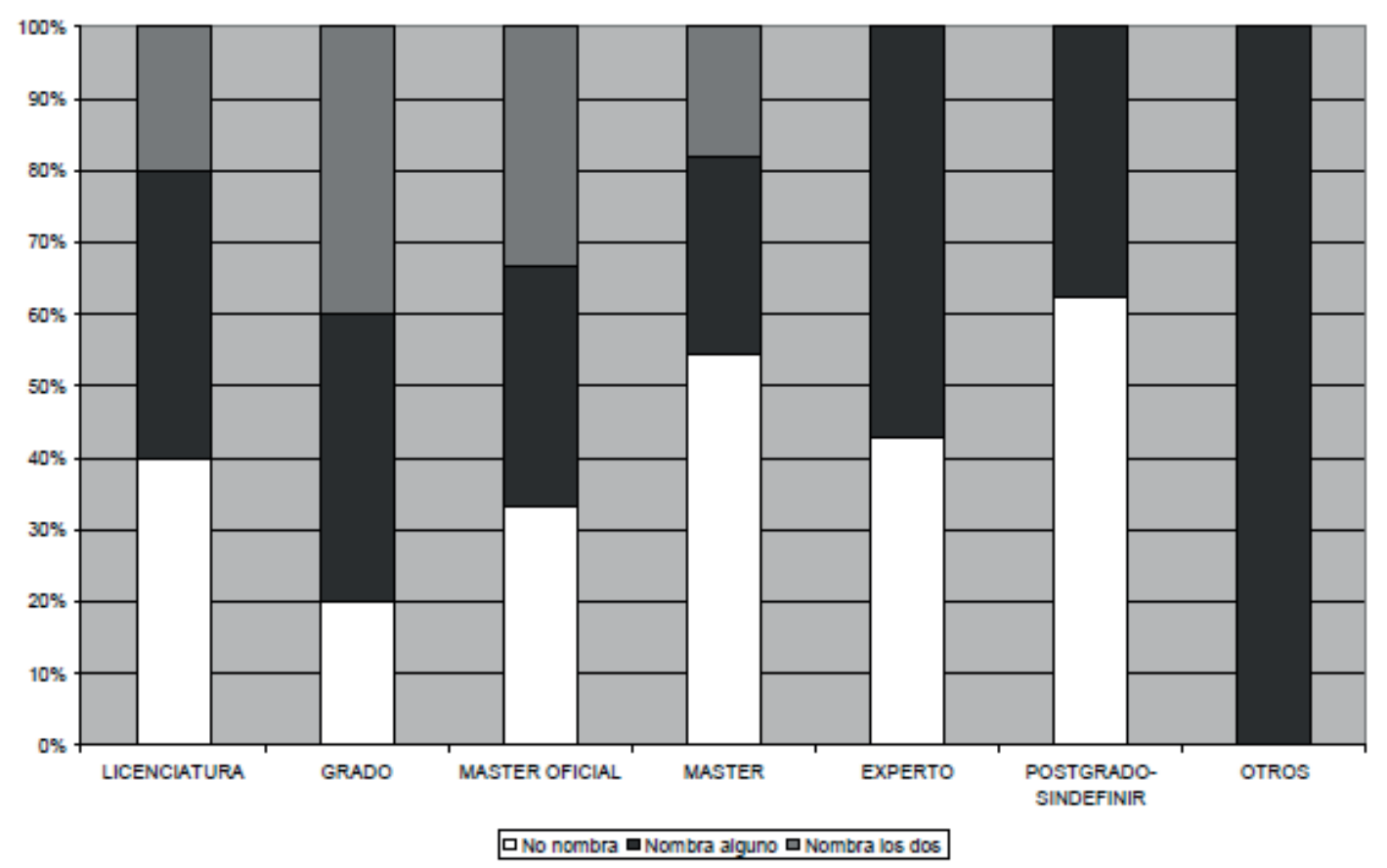

Figura 2. Información sobre los perfiles profesionales según tipo de oferta educativa 


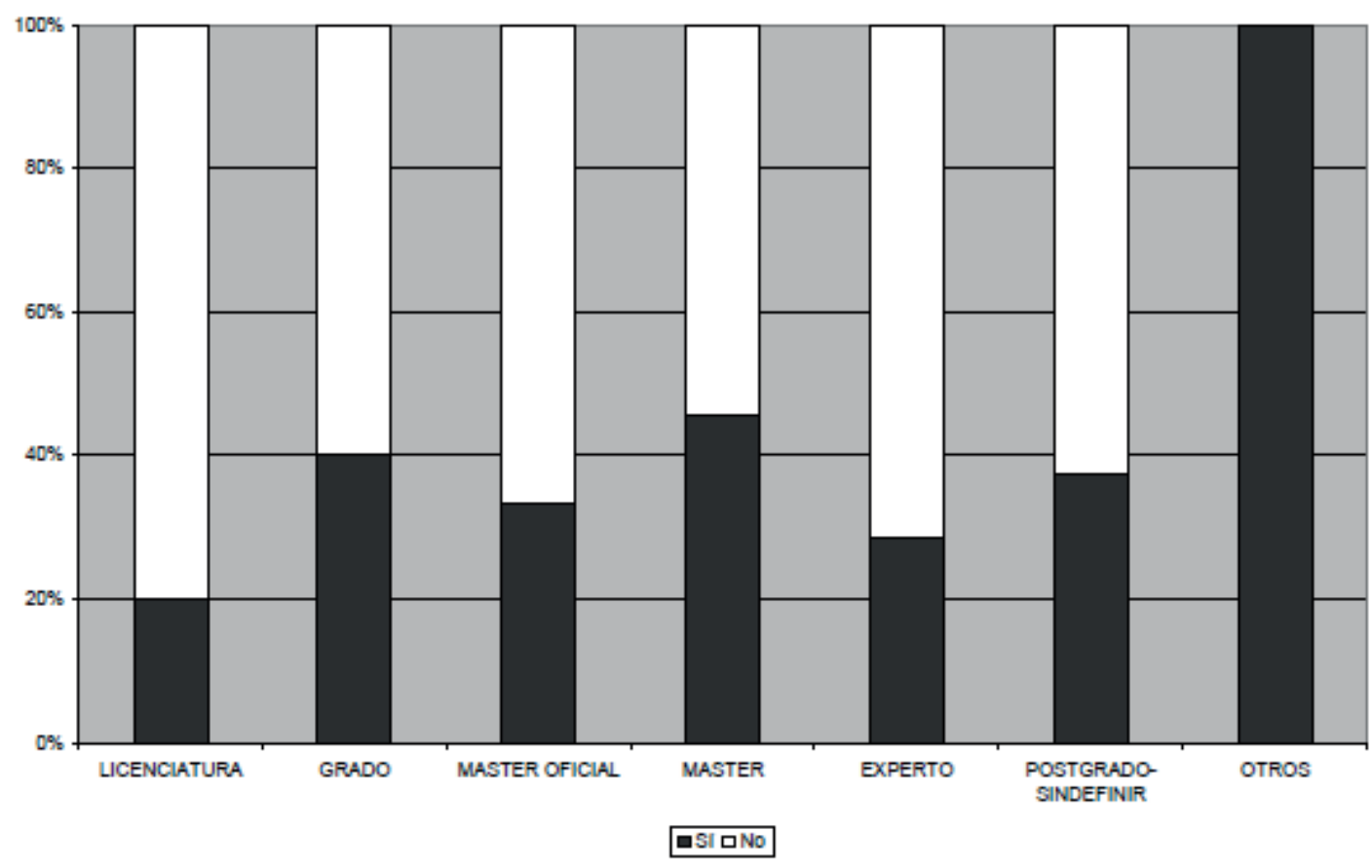

Figura 3. Información sobre los perfiles específicos según tipo de oferta educativa

La ausencia de información sobre las competencias se agudiza en todos los casos. De los 22 casos que nombran los perfiles, sólo el 18\% definen los perfiles generales, y el $14 \%$ incluyen, además competencias. De estos casos, algo superior es el porcentaje que define los perfiles específicos (27\%) pero sigue siendo sólo el 13\% los que incluyen sólo competencias vinculados a los mismos.

En consecuencia, la "mortalidad" de la información en torno a los perfiles específicos es evidente. Sólo 22 casos nombran perfiles, 15 casos nombran los perfiles específicos, sólo 6 los definen y sólo 3 incluyen competencias (El Máster Social Media y Community Manager de la UCM, Experto en economía digital y comunity management de la Rey Juan Carlos y el Master Sociedad de la Información y del Conocimiento de la Oberta de Cataluña).

\subsection{Identificación de las competencias de los perfiles profesionales}

\section{Universidad Complutense de Madrid}

La Facultad de Ciencias de la Información ofrece 9 másters, 3 especialistas, y 7 expertos. De todos ellos, 6 másters y 3 expertos tienen alguna vinculación con los departamentos de comunicación audiovisual y publicidad. Con todo, se han incluido, por su clara especialización en temas de Internet, tres Másters que no cumplirían a priori los criterios de búsqueda: Social Media\&Community Manager. Nuevas Tecnologías en el Mundo Empresarial (se la Facultad de informática), Publicidad 
Interactiva (Centro Superior de Estudios de Gestión de la UCM y IAB) y Comunicación digital (Centro Superior de Estudios de Gestión de la UCM y IAB).

En lo referente a los perfiles profesionales tanto generales como especializados, el grado enumera las salidas profesionales con mayor especialización que las recogidas en el Libro Blanco. Así, menciona hasta nueve salidas profesionales, sin incluir, en ninguna de ellas, el medio online, pues se tratan de perfiles generales.

En cuanto a las capacidades, el grado recopila aquellas relacionadas con los conocimientos a ser adquiridos. Entre ellas se recoge la "creación de nuevos mensajes y nuevos soportes". Por asignatura, ninguna es específica según su denominación. De todas las asignaturas, sólo unas pocas, y de los dos primeros cursos, tienen el temario disponible a través de Internet.

Entre ellas, únicamente "Marketing aplicado a la publicidad" recoge en su temario unidades relacionadas con los medios online. Se prevé que serán más las asignaturas que incluirán estos temas en el grado ya que en los dos últimos años de la licenciatura se introduce en las siguientes tres obligatorias o troncales: Narrativa audiovisual publicitaria, Estructura de la actividad publicidad y Creatividad. Aunque fuera de los criterios de observación inicial, también hay dos optativas que lo mencionan: Los soportes en la comunicación del texto literario e Industrias culturales.

De los nueve títulos propios y cursos ofertados por los departamentos de Comunicación Audiovisual y Publicidad, el master de "Comunicación corporativa y publicitaria" y el de "Comunicación de Instituciones Públicas y Políticas" tienen asignaturas y contenidos similares. Una parte de su temario incluye la comunicación online (y también la publicidad en el primer caso).

El master de "Gestión Publicitaria" presenta dos unidades del temario relacionadas (Internet y Nuevas Tecnologías, y Publicidad 2.0: Social Media, Redes Sociales y Community Manager). En cambio en la información disponible del curso de experto en "Creatividad y Planificación Estratégica" no se hace ninguna mención. El master en tecnologías digitales interactivas también forma parte del temario la "Integración multimedia e Internet". En el resto de cursos no se introduce ninguna unidad ni se nombran perfiles generales ni específicos relacionados.

Los dos masters vinculados al Centro Superior de Estudios de Gestión de la UCM y el de la Facultad de Informática son los más específicos. El master de "Comunicación online" recoge en su plan de estudios la figura del director de Marketing o de Comunicación. Cuando llega a la parte digital incluye aspectos centrados en la gestión de estos medios (Account Planning, estrategia y Planificación de contactos digitales, Dirección de la Comunicación de Digital, Usabilidad \& Web Site Development, Search Engine Marketing \& Digital Monitoring, Social Media Marketing, Creatividad en Comunicación Digital, Nuevos medios digitales \& Mobile Marketing, Planificación de campañas digitales, Online Trenes). 
El master de "Publicidad Interactiva" proporciona más información sobre los contenidos de las asignaturas pero no se centra en ningún perfil concreto. Curiosamente, el que contiene más información es el de la Facultad de Informática. Enumera, también, algunas competencias generales que serán adquiridas tras realizar el master, tales como: "Habilidad para integrarse en un equipo de profesionales donde gestionar la innovación en la empresa o dominio de nuevas tecnologías que están actualmente disponibles para su inmediata aplicación al mundo empresarial" como ejemplo.

\section{Universidad Rey Juan Carlos}

En esta universidad se han analizado un total de 8 tipos de estudios distribuidos de la siguiente forma: los cuatro cursos del grado en Publicidad y Relaciones Públicas, los tres cursos vigentes en la licenciatura de Publicidad y Relaciones públicas (tercero, cuarto y quinto); dos másteres oficiales; tres títulos propios de experto y un curso de formación continua.

Cabe señalar que, a modo de excepción, y pese a estar fuera de los parámetros de búsqueda descritos en el método, se han incluido en el análisis el título propio titulado "Experto en Economía digital y community management" y el curso de formación continua "Marketing 2.0. El nuevo cliente", por citar ambos en sus planes de estudios algunos de los perfiles especializados descritos en el marco teórico. El título propio es ofertado por el departamento de Economía aplicada, que no tiene adscrita docencia en Publicidad y Relaciones Públicas ni está vinculado a este ámbito, y el curso de formación continua no tiene entidad de título propio.

Hecha estas aclaraciones, indicar que es precisamente en estos dos estudios en los únicos en los que se citan los perfiles especializados. Estos perfiles son: "Community manager", "SEO" y "SEM".

En la licenciatura se nombran los perfiles generales, aunque no se les da la denominación ofrecida por el Libro Blanco, sino que se alude a que los licenciados en Publicidad y Relaciones Públicas están capacitados para desarrollar su actividad profesional en funciones tales como la creación, producción, investigación y planificación, gestión, comercialización, y creación de imagen corporativa.

Además, el plan de estudios incorpora un apartado de salidas profesionales en el que se afirma que los Licenciados en Publicidad y Relaciones Públicas estarán especialmente preparados para "desempeñar su profesión en el ámbito de la publicidad interactiva on-line, tanto en portales generales como especializados". No se ha podido acceder al contenido de las asignaturas de la licenciatura a través de la web. 
Con respecto a las capacidades específicas, señalar que aparecen algunos contenidos claramente vinculados a Internet en algunos de los estudios analizados. En el grado, encontramos asignaturas como "Nuevas tecnologías y la sociedad de la información", de formación básica e impartida en primer curso, que incluye los temas: "Sociedad de la Información y concepto de Red", descrito como la creación, trabajo y producción cultural en RED," De las Industrias Culturales a los medios de comunicación en Red" e "Internet y los nuevos medios de comunicación digitales". En tercer curso, se ofertan dos asignaturas cuyos contenidos sirven a nuestro propósito.

La asignatura "Creatividad en la elaboración del mensaje publicitario", que incluye el tema "Elaboración del mensaje según las posibilidades de los medios", descrito como "la interactividad, la viralidad y la conectividad a través de la web 2.0: Redes Sociales, mobile marketing"; y la asignatura obligatoria denominada "Comunicación multimedia". Esta última incluye en su todo su temario contenidos relativos al medio Internet.

También aparecen contenidos vinculados con Internet en el título propio "Experto en Economía digital y community management" y en el curso de formación continua “Marketing 2.0. El nuevo cliente. En el título propio los contenidos se estructuran en dos bloques: "Redes sociales y comunidades virtuales" por un lado, y "Herramientas del Community Manager" por otro.

En el segundo bloque se habla de gestión de contenidos digitales y "Vanity Management": “blogs", “wikis”,y "mashups”. En el curso de formación continua se incluye en los contenidos: "Social Media Marketing", "Reputación Online" e "Identidad Digital”, “Posicionamiento SEO y SEM" y “Community Manager”.

\section{Universidad de Valladolid}

Los estudios analizados han sido de dos tipos: todos los cursos del grado en Publicidad y Relaciones Públicas y los cursos vigentes de la licenciatura en Publicidad y Relaciones Públicas (tercero, cuarto y quinto).

En lo referente a los perfiles profesionales tanto generales como especializados, en el grado no aparece ninguna información en este sentido, mientras que en la licenciatura se nombran las salidas profesionales, haciendo alusión, a través de la descripción de tareas, a los perfiles generales.

Estas tareas son, para el área de la publicidad: desarrollo artístico y creativo de piezas publicitarias, selección de los medios de comunicación, relación con los clientes (asesoramiento y planificación de campañas, ejecución y seguimiento); para el área de las Relaciones Públicas: convenciones, congresos, publicaciones, organización de acontecimientos, actos protocolarios y servicios de prensa. 
En cuanto a las competencias que podrían ser propias de los perfiles específicos, dentro de la licenciatura sólo hay una asignatura en cuyo temario se refleja una alusión al medio Internet, se trata de Fundamentos de las Relaciones Públicas, ofertada como complementos de formación. En esta asignatura se imparte un tema denominado "Relaciones públicas e Internet", cuyo contenido aparece detallado de la siguiente forma: "Creación y gestión con éxito de un sitio web; promoción de productos en Internet; relaciones con inversores y clientes, las intranets y las extranets".

En el primer curso del grado hay dos asignaturas, una de formación básica denominada "Comunicación, educación y sociedad en el contexto digital" y otra obligatoria cuyos contenidos incluyen conceptos vinculados a Internet tales como "redes sociales", "blogs" y "publicidad en Internet". Hay también una asignatura de carácter optativo denominada "Creatividad on line", que no incluye temario.

\section{Universidad de Alicante}

Se han analizado 6 tipos de estudios: dos cursos $\left(3^{\circ}\right.$ y $\left.4^{\circ}\right)$ vigentes de la licenciatura en Publicidad y Relaciones Públicas, todos los cursos del grado en Publicidad y Relaciones Públicas, un máster oficial y tres títulos de especialistas. También existe un máster en comunicación online que está formado por dos de esos tres títulos mencionados.

En cuanto a la licenciatura, se nombran perfiles generales y competencias pero incluidas en la información general y sin hacer mención a Internet o a las nuevas tecnologías. El plan de estudios es del año 2002, anterior al Libro Blanco, por lo que se comprende que dichas definiciones no fueran incorporadas. En cuanto a perfiles específicos, en la titulación, sólo queda recogido el webmaster en creatividad. Otras asignaturas introducen contenidos relacionados con Internet (elaboración de textos publicitarios y derecho de la publicidad) pero no incluyen perfiles.

El grado recoge los perfiles de la ANECA y sus definiciones. Además menciona una competencia que pudiera estar relacionada: "E37: habilidades para la innovación y desarrollo de nuevos medios y soportes". En esta ocasión, más asignaturas incorporan competencias y contenidos relacionados con Internet y con la publicidad online.

Hasta 18 asignaturas incorporan la utilización de las nuevas tecnologías como capacidad instrumental. Como parte de los contenidos (competencias cognitivas): Semiótica de la comunicación de masas (conocer las implicaciones de las nuevas tecnologías sobre la comunicación y su impacto también sobre los medios de comunicación de masas clásicos); Comunicación corporativa (Habilidades para la innovación y desarrollo de nuevos medios y soportes); Imagen corporativa (Adaptar la comunicación a los nuevos medios o soportes surgidos incesantemente en la sociedad de la tecnología de la información); Gestión de la información en comunicación (Desarrollo de dinámicas de comunicación de los nuevos medios en 
Internet y prever estrategias de adaptación a nuevos contextos en la comunicación); Comunicación y Medios escritos (incluye en el descriptor "Estudio y capacitación en la tecnología, el análisis y la expresión en formas y medios impresos y electrónicos"; Comunicación y medios audiovisuales (Estudio de la comunicación e información escrita, audiovisual y digital como marco de referencia); Investigación y planificación de medios (Habilidades para la innovación y desarrollo de nuevos medios y soportes; Conocer las tendencias en la difusión de la comunicación publicitaria, soportes y formatos, con especial atención a la comunicación a través de Internet).

El Máster Oficial se dirige a formar gestores y directores de la comunicación. Por lo que atiende a un perfil muy genérico. Tiene un itinerario que trata de orientar a posibles investigadores que pretendan continuar con el doctorado.

Los perfiles más específicos son marcados por los cursos de especialistas.

Se trata de un curso relacionado con el diseño y la creación de videojuegos que podría representar, aunque realmente no es su propósito, el advergaming, otro curso de diseño web, no recogido en los perfiles que se han incluido y, finalmente, el de "Social Media y Community Management" que se centra en los medios sociales, aunque tiene un módulo complementario que trata de proporcionar otras técnicas representadas por los perfiles objeto de análisis (SEO, SEM, planificadores, móvil...).

\section{Universidad Oberta de Cataluña}

Se han analizado 9 tipos de estudios: los dos cursos ofertados de la licenciatura en Publicidad y Relaciones Públicas, todos los cursos del grado en Comunicación, un máster oficial de doctorado y 6 cursos de posgrado.

En lo referente a la licenciatura, con respecto a los perfiles profesionales no se dispone de información. En materia de competencias, cita el medio Internet en dos de asignaturas troncales, en "Planificación y medios publicitarios I", concretamente incluye un módulo denominado "Internet y los medios interactivos: modalidades y características"; y en "Teoría y Técnica de las Relaciones Públicas II" se incluye el módulo "Relaciones públicas en sistemas interactivos multimedia".

La Universitat Oberta de Cataluña, a diferencia del resto de Universidades analizadas, oferta un Grado en Comunicación, que se caracteriza por integrar competencias propias del área de Periodismo, del área de Publicidad y Relaciones Públicas, y del área de Comunicación Audiovisual. Los perfiles profesionales mencionados en este grado son los generales, y no los especializados.

Así, de entre todos los perfiles nombrados aquellos que se insertan dentro del ámbito propio de la Publicidad y las Relaciones públicas serían: Dircom, director de relaciones públicas, consultor de comunicación corporativa, director de protocolo y relaciones institucionales, creativo publicitario; director de cuentas, planificador de medios y planner (planificador estratégico). Las competencias que se describen, al igual que 
sucede con los perfiles son competencias generales, de manera que no se alude al medio Internet en ninguna de ellas.

El máster es un programa de doctorado que lleva por título "Sociedad de la información y el conocimiento", y que es ofertado por el Instituto de investigación 14 "Internet Interdisciplinary Institute" (IN3). El IN3 está especializado en investigación sobre la sociedad en red y la economía del conocimiento, así como en el estudio de las tecnologías de red y áreas específicas de software (Universitat Oberta de Cataluña. IN3).

En este máster, dadas su especificidad, sólo se nombran y describen perfiles profesionales especializados y competencias específicas, pero enfocadas hacia la titulación de Informática (ie. especialidad de Seguridad en redes y sistemas; de Seguridad en servicios y aplicaciones; de Gestión y auditoria de la seguridad informática). Con respecto a las competencias, las que más se acercan al medio Internet se incluyen en asignaturas optativas ${ }^{15}$.

Los cursos de postgrado a destacar son cinco:

Postgrado "Dirección en comunicación": Sólo contiene información relativa a las competencias específicas a modo de contenido de una de las asignaturas ofertadas. Se trata de "Marketing directo e interactivo", e incluye las características de las comunicaciones en la World Wide Web e Internet, experiencias de flujo en línea y el sitio web.

\footnotetext{
${ }^{15}$ Los media en la sociedad de la información (Entender la interrelación entre los nuevos media y la sociedad de la información; Introducir a los estudiantes en diferentes perspectivas teóricas que los ayuden a construir un marco conceptual necesario y propio para entender los diferentes aspectos que definen a los nuevos medios y a sus formas cambiantes; Reflexionar sobre los nuevos usos emergentes de los medios de comunicación en la Sociedad de la Información (económicos, políticos, coercitivos y simbólicos); Capacitar para analizar casos concretos en cada uno de los usos de los nuevos medios de comunicación en red Analizar la interrelación entre el desarrollo contemporáneo de los medios de comunicación y las transformaciones sociales de la Sociedad de la Información) ; Globalización, pluralismo legal e Internet (Comprender el alcance que la irrupción de la Web Semántica está produciendo en Internet y sus posibles efectos en las organizaciones sociales y en la vida cotidiana; Conocer los efectos de las TIC en el mundo del derecho: la digitalización de la justicia y la aparición de nuevas formas alternativas de resolución de los conflictos); Marketing y comercio electrónico (Entender la importancia de que las iniciativas de negocio en la red se orienten estratégicamente hacia el mercado, el marketing y el establecimiento de relaciones continuadas con los clientes; Comprender las principales líneas que orientan a las empresas en el diseño de estrategias de marketing electrónico; Conocer el impacto de Internet en el desarrollo de procesos de investigación de mercado; Comprender de qué modo Internet puede aumentar el valor que se proporciona al consumidor; Ser capaces de cuestionaros y reflexionar si Internet es un espacio de intercambio más competitivo y eficiente; Conocer y diseñar estrategias de precios y marcas en Internet; Adquirir capacidad analítica en el estudio de los procesos de comunicación comercial en Internet; Adquirir los conocimientos y las capacidades analíticas en torno a las transformaciones de la comunicación publicitaria que se derivan del uso de Internet como soporte en la comunicación; Identificar y comprender las ventajas competitivas de la venta en línea en comparación con otros formatos comerciales).
} 
Postgrado "Plataformas y tecnologías multimedia": En lo relativo a los perfiles profesionales este postgrado indica que, desde un punto de vista disciplinar, se puede considerar al especialista en multimedia como un profesional especializado en roles como guionización interactiva, animación, vídeo digital, comunicación visual, programación de autor y web, sonido, dirección y gestión, diseño y edición web, entre otros. En la asignatura "Nuevos espacios y entornos interactivos" se incluyen los contenidos Marketing y publicidad interactiva; portales y sitios web.

Postgrado "Publicidad en Internet y nuevos medios digitales": La información analizada de este postrado se centra sólo en contenidos. Así, hallamos dos módulos teóricos que se ajustan al objetivo de nuestro estudio. Uno denominado "Estrategia Creativa" donde se incluye: La nueva publicidad nace con la aparición de Internet; Cómo plantear estrategias para una campaña integral que incorpora Internet o medios digitales; Cómo plantear estrategias para una campaña en línea. Y otro denominado "Creatividad aplicada" en el que se desglosan los siguientes contenidos: ¿En qué es diferente la creatividad aplicada a los medios digitales?; La Web: la base de Internet y de la mayoría de los medios digitales; Los formatos publicitarios dentro y fuera de una web: banners, minisites y pop-ups; Otros formatos publicitarios fuera de la web: emailing y campañas virales; Las posibilidades publicitarias de la web 2.0: blogs, redes sociales y comunidades.

Postgrado "Redes sociales e intercambio de conocimiento": Incluye información relativa a contenidos dentro de la asignatura "Inteligencia e identidad colectiva en las organizaciones": Inteligencia competitiva a través de las redes sociales; Identidad y representación corporativa.

Postgrado "Media\&Web Impact: estrategias de visibilidad y audiencia": Referente a perfiles profesionales especializados, el postgrado en su presentación señala que está dirigido a los profesionales del ámbito de la información y comunicación que trabajen en medios de comunicación, marketing, agencias de planificación de medios, agencias publicitarias y empresas de diseño de entornos web, como webmasters.

En cuanto a los contenidos específicos por asignaturas, en la asignatura "Audiencia y visibilidad en los medios" se detalla: la medida y el estudio del impacto, la visibilidad y la audiencia en los medios de comunicación: prensa, TV, radio y Web; en la asignatura "Web Impact I: Buscadores": Funcionamiento de un buscador, software de relevancia, motor de búsqueda (buscadores generalistas, buscadores especializados, metabuscadores); y por último en la asignatura "Web Impact II: calidad de contenidos": Indicadores generales (autoría, objetivos del sitio web, acceso a la información), indicadores específicos, indicadores de calidad en la prensa digital e indicadores de la Web 2.0. 


\section{CONCLUSIONES}

Los grados son la oferta educativa que en mayor medida incluye en sus contenidos la exposición de perfiles profesionales, sean estos generales o específicos vinculados a Internet. Se advierte, así, la influencia de la reforma universitaria.

No obstante, los contenidos y perfiles más específicos son abordados sobre todo por medio de los títulos propios, a través de másters, postgrados o cursos. En contradicción, este tipo de estudios, vinculados a un departamento de comunicación, tienen en su web menos información. Por ejemplo, existe menos menciones al sector y no aparecen los temarios de las asignaturas.

La imposibilidad de acceder a los contenidos de las asignaturas a través de la web supone una limitación a este estudio, al no disponer de esta información en abierto. Además, pese a que las competencias profesionales son el elemento fundamental desde la última reforma universitaria (en todo tipo de estudios aunque más en esta clase de oferta) la ausencia de las mismas en la información proporcionada es evidente.

Por tanto, con la información existente, no se puede identificar competencias específicas propiamente dichas, aunque sí se advierte que el medio online suele ser incluido en los temarios de las asignaturas de marketing, creatividad, relacionadas con la codificación del mensaje, las relaciones públicas y los medios. Se refleja, así, la tendencia que se observa en el mercado.

Los especialistas en Marketing, Publicidad y Relaciones Públicas podrían implementar las técnicas de comunicación en las que son expertos en este medio pero deben conocer las específicas para trabajar con el mismo. En este sentido, y en concreto, todas las universidades analizadas incluyen en sus contenidos específicos como mínimo un tema o bloque relacionados con las redes sociales. Esto refleja la importancia de las mismas como medio de sociabilidad online.

En consecuencia, existe un interés en la especialización online y/o en los temas relacionados con Internet en las universidades analizadas. En todas ellas hay títulos propios y/o asignaturas que incluyen contenidos sobre Internet como medio de comunicación. No obstante, según la información facilitada en su web, destaca la poca oferta de la UVA.

Pese a este interés por ofertar estudios existen carencias de información sobre el sector, los perfiles y las competencias a través de la web, lo que no deja de ser una contradicción. Igualmente, y como cuestión sobre la que se debe reflexionar desde la publicidad y las relaciones públicas, destaca la oferta específica de cursos relacionados con algunos de los perfiles mencionados (CM, SEO/SEM) de departamentos distintos al área de Comunicación Audiovisual y Publicidad, tales como la economía o la informática. 
Este hecho podría obedecer a que los conocimientos vinculados a Internet están todavía en "tierra de nadie", lo cual tiene su claro reflejo en el eclecticismo de los estudios en los que éstos aparecen. Dado que se trata en su mayoría de títulos propios, este hecho también podría responder a la oportunidad de ofrecer un producto atractivo para el estudiante, quien debe desembolsar una importante suma en concepto de matrícula para adquirir la formación ajustada a las demandas actuales de un mercado al que accederá en plena crisis.

En cualquier caso, la sociabilidad de Internet se vislumbra como el aspecto más valorado por los académicos de las Universidades a la hora programar sus asignaturas y fijar los objetivos de aprendizaje, lo que pone de manifiesto el creciente interés en este medio como instrumento de comunicación social. En este sentido, Manuel Castells (2000) considera a Internet un canal flexible en contraposición a las organizaciones estructuradas, con gran capacidad para movilizar a grandes grupos sociales en torno a un tema o problema social, y con repercusión mundial.

La capacidad de Internet, por tanto, se presupone muy poderosa, pero lo cierto es que aún hoy no es conocida en toda su amplitud, por lo que debería ser objeto de estudio por parte de los investigadores sociales.

\section{REFERENCIAS}

AERCO. (2009). La función del Community Manager. Madrid: Autor.

AIMC. (2011). Resumen general EGM, de octubre 2010 a mayo 2011. Madrid: Autor.

ANECA. (2004). Libro Blanco. Títulos de Grado en Ciencias de la Información. Madrid: Autor.

Castells, M. (2000). Internet y la Sociedad en red. Conferencia de Presentación del Programa de Doctorado sobre la Sociedad de la Información y el Conocimiento. Universitat Oberta de Catalunya.

González, J. \& Wagenaar, R. (2003). Tuning Educational Structures in Europe. Informe Final. Fase Uno. Universidad de Deusto y Universidad de Groningen. Bilbao.

IAB-SPAIN. (2010). Observatorio del mercado laboral de los profesionales del marketing, la comunicación y la publicidad digital. $1^{a}$ Oleada. Recuperado el 20 de junio de 2011, de http:/ / www.iabspain.net/ver.php?mod=noticias\&identificador=100.

IAB-SPAIN. (2011). Observatorio del mercado laboral de los profesionales del marketing, la comunicación y la publicidad digital. $2^{a}$ Oleada. Recuperado el 20 de junio de 2011, de http:/ / www.iabspain.net/ver.php? $\bmod =$ noticias\&identificador=100. 
INFOADEX. (2008). Resumen. Estudio infoadex de la inversión publicitaria en España 2008. Madrid: Autor.

INFOADEX. (2011). La inversión publicitaria gestionada en 2010 por las agencias de publicidad y las agencias de medios. Madrid: Autor.

Kaplan, A. M. \& Haenlein, M. (2010). Users of the world, unite! The challenges and opportunities of social media. Business Horizons, 53(1): 59-68.

MEC. (2007). Real Decreto 1393/2007, de 29 de octubre, por el que se establece la ordenación de las enseñanzas universitarias oficiales. BOE, 260: 44037-44048.

MECD. (2003). La integración del sistema universitario español en el espacio europeo de enseñanza superior. Documento- Marco. Recuperado el 20/05/2011, de http.//www.eees.ua.es/conferencias/Documento-Marco_10_Febrero.doc.

TCANALYSIS (2011): El uso de las redes sociales en España. Recuperado el 20 de mayo de 2011, de http://www.tcanalysis.com/2011/03/22/infografia-sobre-el-uso-de-lasredes-sociales-en-espana/.

\section{Natalia Papí-Gálvez}

Doctora por la Universidad de Alicante (UA) en 2004 y Licenciada en Sociología con la especialidad en Marketing y Publicidad (UA) en 1999, ambos con premio extraordinario. Obtuvo una beca de investigación por la Dirección General de la Dona (1999) y posteriormente disfrutó de la beca FPI nacional en la Facultad de Ciencias de la Información de la Universidad Complutense Madrid. Investigadora especializada en estudios de género y en evaluación de la comunicación. Ha dirigido y colaborado investigaciones del Plan Nacional relacionadas con estas líneas de trabajo. Profesora titular en el Departamento de Comunicación y Psicología Social (UA). Docencia en la Licenciatura de Publicidad y Relaciones Públicas. Directora del grupo de investigación “Grupo de estudios de comunicación estratégica (E-COM)" de la Universidad de Alicante.

\section{Sonia López-Berna}

Licenciada en Sociología con la especialidad de Marketing y Publicidad por la Universidad de Alicante. En el año 2004, obtuvo el DEA en el Programa de Doctorado de Bienestar Social y Desigualdades ofertado por esta Universidad. En la actualidad se encuentra realizando su tesis doctoral. La línea de investigación de su tesis está encaminada al estudio de la profesión del publicitario, y se halla enmarcada en la Sociología de las Profesiones. Ha publicado varios capítulos de libro y artículos dentro en este ámbito. Pertenece al grupo de investigación "Grupo de estudios de comunicación estratégica (E-COM)" de la Universidad de Alicante. Es profesora colaborador en el grado y en la licenciatura en Publicidad y Relaciones Públicas de la Universidad de Alicante. También es profesora tutora de prácticas en empresa de la licenciatura en Publicidad v Relaciones Públicas de la Universidad de Alicante. 